\section{P274 \\ DELIBERATE PRACTICE MAKES PERFECT: REGULAR 30 MIN LOW-FI SIMULATION-BASED RESUSCITATION PRACTICE IMPROVES NCHD CONFIDENCE, TECHNICAL AND NON-TECHNICAL SKILLS IN PAEDIATRIC EMERGENCIES}

Heather Cary*, Irena Christol. Sligo University Hospital, Sligo, Ireland

\subsection{6/archdischild-2019-epa.624}

Introduction Simulation is a method of teaching and continuing education that is particularly well suited to fields that experience infrequent but high-stakes events, of which paediatric medicine is an excellent example. In this study, we created a simulation-based multidisciplinary continuing education intervention and measured its effect on paediatric NCHD's perceived performance in the areas of technical and nontechnical skills as well as confidence, self-efficacy and participant satisfaction.

Aims \& objectives The aim of the project was to determine if frequent practice of paediatric emergency situations with lowfi mannequins and a tablet based 'monitor' improves staff knowledge, technical and nontechnical skills as well as confidence and self-efficacy.

Methodology The intervention took the form of weekly planned $30 \mathrm{~min}$ teaching sessions that rotated between the Paediatric ward, Emergency Department, and Maternity ward. The format of these teaching sessions was immersive simulation in the form of APLS or NRP moulages using simple plastic mannequins and a tablet monitor controlled by the examiner's phone.

The effects of the intervention were measured using a pre- and post-intervention questionnaires assessing paediatric NCHD's perceived performance in the areas of technical skills (e.g. intubation) and non-technical skills (e.g. situational awareness) as well as confidence and self-efficacy. The questionnaires also addressed attitudes to simulation in general and this intervention in particular (ie participant satisfaction). Pre-intervention $n=5$, post-intervention $n=10$; scores were compared using Wilcoxin Signed-Ranks.

Results There was no difference between pre and post intervention attitudes to the importance of knowledge, technical and non-technical skills, and the opportunity for practice however these were all very highly rated prior to the intervention. No significant difference was found between pre and post intervention self-rated confidence, technical, nontechnical skills, nor was there a difference in self-rated performance in the most recent resuscitation performed. $100 \%$ of participants either agreed or strongly agreed that the intervention improved their clinical knowledge, situation awareness, team working, communication and confidence. $89 \%$ of participants either agreed or strongly agreed that the intervention improved their technical and nontechnical skills, decision making and leadership. The most frequently occurring negative feedback in $89 \%$ of participants was that they would have liked more frequent simulation sessions.

Conclusion It is likely the small sample size and reliance on non-parametric tests contributed to the lack of significant results. The intervention was highly popular with participants who described a subjective improvement in clinical knowledge, confidence, situational awareness and technical and nontechnical skills following the simulation sessions.

\section{P275 \\ AN AUDIT OF DOCUMENTATION AND OUTCOMES IN PAEDIATRIC EMERGENCY DEPARTMENT PRESENTATIONS OF LOWER RESPIRATORY TRACT INFECTIONS}

${ }^{1}$ Allison Clark*, ${ }^{2}$ Turlough Bolger. ${ }^{1}$ Trinity College Dublin, Dublin, Ireland; ${ }^{2}$ Paediatric Emergency Deparment, National Children's Hospital, Dublin, Ireland

10.1136/archdischild-2019-epa.625

Introduction Lower respiratory infections are a common Paediatric ED presentation. It is important to use resources efficiently in the ED to ensure that those children requiring admission or discharge can be readily identified. We studied the documentation in the medical notes of history, examination and risk factors to assess whether this resulted in appropriate outcomes for patients.

Methods We performed a sequential audit of lower respiratory tract infection presentations to a Paediatric ED using our electronic patient record. We identified 333 presentations over a 6 month period. This includes 316 different patients, as 17 different patients had multiple presentations to the ED for similar symptoms and were subsequently diagnosed with LRTI. Results Symptoms were evident for an average of 7.48 days (0.5 to 120 days). Patients referred to ED by a doctor in $58 \%$ (194/333). Prior treatment, such as inhalers or antibiotic medications was given in $51 \%$ of cases (171/333). Risk factors identified for LRTI included history of asthma (55/333), previous admission for a respiratory infection (84/333), preterm birth less than 34 weeks gestation (8/333), previous invasive positive pressure ventilation (10/333), developmental delay/ PEG feedings (8/333), and history of immunodeficiency disease (0/333).

Documentation of history of presenting symptoms consistent with LRTI were cough (315/333), fever (235/333), and sputum (98/333).

Documentation of the examination findings included respiratory rate only $79 \%$ (264/333), oxygen saturation only $49 \%$ $(162 / 333)$, and evidence of work of breathing in $86 \%$ (296/ 333). $251(75 \%)$ chest $x$-rays were completed while in the ED.

Overall, 126/333 patients (38\%) were admitted. Looking at subgroups who should predict admission, 74/194 (38\%) of GP referrals, those requiring x-ray 112/251 (44\%), those who had both fever and sputum present 19/52 (37\%), and short duration of symptoms 109/207 (40\%) required admission.

Discussion Our documentation of history and examination was generally poor ranging from 49-95\%. None of the examined admission risk factors predicted admission suggesting that the clinical formulation and utilisation of resources was haphazard.

\section{P276 THREE CASES OF COMPLICATED BACTERIAL SINUSITIS IN OLDER CHILDREN}

Laura Reaney*, Nandini Kandamany. Temple Street Children's University Hospital, Dublin 1, Ireland

\subsection{6/archdischild-2019-epa.626}

Aims The paranasal sinuses are a common site of infection in children and adolescents. While these are usually viral respiratory infections, a small percentage can be complicated by secondary bacterial infection which can give rise to potentially serious complications. Three cases of complicated bacterial 